\title{
Fluxbranes in string theory
}

\section{Citation}

Gutperle, Michael, and Andrew Strominger. 2001. "Fluxbranes in String Theory." Journal of High Energy Physics 2001 (6): 035-035. https://doi.org/10.1088/1126-6708/2001/06/035.

\section{Permanent link}

http://nrs.harvard.edu/urn-3:HUL.InstRepos:41417260

\section{Terms of Use}

This article was downloaded from Harvard University's DASH repository, and is made available under the terms and conditions applicable to Other Posted Material, as set forth at http:// nrs.harvard.edu/urn-3:HUL.InstRepos:dash.current.terms-of-use\#LAA

\section{Share Your Story}

The Harvard community has made this article openly available.

Please share how this access benefits you. Submit a story.

Accessibility 
hep-th/0104136

HUTP-01/A018

\title{
Fluxbranes in String Theory
}

\author{
Michael Gutperle and Andrew Strominger \\ Jefferson Physical Laboratory \\ Harvard University \\ Cambridge, MA 02138
}

\begin{abstract}
A flux p-brane in $\mathrm{D}$ dimensions has $(\mathrm{p}+1)$-dimensional Poincare invariance and a nonzero rank (D-p-1) field strength tangent to the transverse dimensions. We find a family of such solutions in string theory and M-theory and investigate their properties.
\end{abstract}

April 2001 


\section{Contents}

1. Introduction . . . . . . . . . . . . . . . . . . . . . . . . . . . . . . . . 1

2. The IIA F7-brane . . . . . . . . . . . . . . . . . . . . . . . . . . . . . . . 3

2.1. The Solution . . . . . . . . . . . . . . . . . . . . . . . . . . 4

2.2. $0 \mathrm{~A} \leftrightarrow \mathrm{IIA}$ Duality . . . . . . . . . . . . . . . . . . . . . . . . . . . . . . 5

2.3. The Fate of the Tachyon . . . . . . . . . . . . . . . . . . . . . . . . . . 6

2.4. F7 $\leftrightarrow$ IIA Cone Duality . . . . . . . . . . . . . . . . . . . . . . . . . . . 7

3. ALE $\leftrightarrow$ Supersymmetric F5-brane Duality . . . . . . . . . . . . . . . . . . . . 9

4. The General Fp-brane Solution . . . . . . . . . . . . . . . . . . . . . . . . . 10

4.1. The Attractor Solutions . . . . . . . . . . . . . . . . . . . . . . . . . . . 12

4.2. The M-Theory F6-brane . . . . . . . . . . . . . . . . . . . . . . . . . . 13

4.3. The M-Theory F3-brane . . . . . . . . . . . . . . . . . . . . . . . . . . 14

4.4. Type II fluxbranes . . . . . . . . . . . . . . . . . . . . . . . . . . . . . 15

5. Critical D(p-1)-branes in Fp-branes . . . . . . . . . . . . . . . . . . . . . . . 17

5.1. The Critical M2-brane in a F3-brane . . . . . . . . . . . . . . . . . . . . . 17

5.2. The Critical M5-brane in a F6-brane . . . . . . . . . . . . . . . . . . . . . . . . 20

5.3. The Critical Dp-brane in a $\mathrm{F}(\mathrm{p}+1)$-brane . . . . . . . . . . . . . . . . . . . 21

6. A Holographic Dual? . . . . . . . . . . . . . . . . . . . . . . . . . . . 23

7. Flux Periodicity . . . . . . . . . . . . . . . . . . . . . . . . . . . . 25

\section{Introduction}

A magnetic monopole is characterized by the integral of the field strength $F$ over the two sphere surrounding the monopole. A magnetic fluxtube, in contrast, is characterized by the integral of $F$ over the transverse plane. The much-studied p-branes of string theory are the generalizations of magnetic monopoles to higher rank field strengths and dimensions. The present work concerns the much less-studied generalizations of magnetic fluxtubes to higher rank and dimensions, which are referred to as fluxbranes or Fp-branes.1 We shall find solutions of this type for all $p<8$ in string theory, as well as for $p=3,6$ in Mtheory. These appear to be interesting family of (in general non-supersymmetric) solutions of string theory. Several duality relations involving the RR two-form field strength will be

1 To be precise an Fp-brane in D-dimensions has $I S O(p, 1) \times S O(D-p-1)$ symmetry and a nonzero rank $(D-p-1)$ field strength, dual field strength, or wedge product of field strengths tangent to the transverse dimensions. 
discussed. These are perhaps part of a larger web of dualities involving all rank forms and Fp-branes which remains to be found.

The classic example of a fluxbrane with gravity is the Melvin universe [1], which is a F1-brane in 3+1 Einstein-Maxwell gravity (and can be embedded in string theory). The solution is

$$
\begin{aligned}
d s^{2} & =\left(1+\frac{B^{2} r^{2}}{4}\right)^{2}\left(-d t^{2}+d z^{2}+d r^{2}\right)+\frac{r^{2}}{\left(1+\frac{B^{2} r^{2}}{4}\right)^{2}} d \phi^{2}, \\
F & =\frac{B r d r \wedge d \phi}{\left(1+\frac{B^{2} r^{2}}{4}\right)^{2}} .
\end{aligned}
$$

$B$ here is the magnetic field strength along the axis $r=0$. The total magnetic flux is

$$
\frac{1}{4 \pi} \int_{R^{2}} F=\frac{1}{B} .
$$

This is finite, so magnetic flux is in a sense confined by gravity. At large $r$, the orbits of $\phi$ become small and the transverse space resembles the surface of a teardrop with an infinite tail.

In string theory one encounters dilatonic generalizations of this solution [2,3, 团. The simplest of these is the IIA F7-brane, which is considered in section 2. In 2.1 we review the M-theory description of the F7 which is simply an identification of flat $R^{11}$ involving a rotation [4, 5,:60. This description strongly suggests a surprising periodicity in the field strength, as well as a dual relation to $0 \mathrm{~A}[7,[8]$ described in 2.2. This is used to motivate a conjecture in subsection 2.3 that the endpoint of $0 \mathrm{~A}$ tachyon condensation is the IIA vacuum. Intriguing similarities between this picture of 0A closed string tachyon condensation and $D-\bar{D}$ open string tachyon condensation are pointed out. In section 2.4 we derive a dual relation of the F7-brane to the IIA theory on a cone by making a new choice for the M-theory circle. In section 3 we consider the supersymmetric IIA F5-brane which is characterized by a nonzero integral of $F \wedge F$ over the transverse $R^{4}$. This also has a flat M-theory lift. We show, by making a new choice of the M-theory circle, that this is dual to IIA theory on an $A_{N}$ ALE space. 
This beautiful duality story for the F7-brane was possible to uncover because of its simple M-theory lift. It is natural to expect that the other Fp-branes (which are in some cases related by T-duality [9]) are also part of an as-yet-uncovered web of dualities. The rest of the paper contains some preliminary investigations of the $p<7$ case. In section 4 we turn to the general Fp-brane solutions in $\mathrm{M}$ and string theory. These differ qualitatively from the IIA F7 in that the flux is not confined and the integral of the field strength over the transverse dimensions does not converge. The solutions can be found analytically only in the special case that the field strength $F$ at the origin goes to infinity (but is finite everywhere else). These analytic solutions have a warped cone-like structure with a singularity at the origin. The singularity is resolved, but the asymptotic behavior unchanged, by making $F$ finite at the origin. We find the solutions perturbatively both near the origin and at infinity and then match numerically. This is less difficult than it might sound because the asymptotic solution is a universal attractor for all regular initial conditions at the origin. In section 5 we consider a special type of excitation of the Fp-brane, namely static but unstable $\mathrm{D}(\mathrm{p}-1)$-brane bubbles. These are analogs of the open string dipoles in an electric field. We find a critical scaling limit in which the string mass is taken to infinity while the radius of and gauge coupling on the D-brane bubble is kept finite. Formally this is a field theory limit. In section 6 we speculate on a possible holographic dual for the Fp-brane. In section 7 we ask whether or not there could be flux periodicity for $p<7$. Some evidence is provided for the case of the Melvin universe from the properties of black hole pair production.

\section{The IIA F7-brane}

In this section we discuss the IIA F7-brane. This case is characterized by a nonzero but finite integral of the RR two form field strength over the two dimensions transverse to the brane. 


\subsection{The Solution}

The simplest way to describe the IIA F7-brane is in terms of its lift to $M$-theory [6]. The reduction from 11 to 10 dimensions involves the usual shift identification, accompanied by a spatial rotation 2 of the angle $\phi$ in the plane transverse to the F7-brane

$$
\begin{aligned}
x^{11} & \sim x^{11}+2 \pi n_{1} R, \\
\phi & \sim \phi+2 \pi n_{2}+2 \pi n_{1} B R^{2} .
\end{aligned}
$$

In other words the dimensional reduction is performed along orbits of the Killing vector $q=\partial_{x_{11}}+B R \partial \phi[5,6]$. The factors of $R$ in the second equation have been chosen for later convenience. In order to perform the Kaluza-Klein reduction to ten dimensions it is convenient to introduce the coordinate $\tilde{\phi}=\phi-B R x^{11}$ which is canonically identified and constant along orbits of $q$,

$$
\begin{aligned}
x^{11} & \sim x^{11}+2 \pi n_{1} R, \\
\tilde{\phi} & \sim \tilde{\phi}+2 \pi n_{2} .
\end{aligned}
$$

In terms of $\tilde{\phi}$ the eleven dimensional metric is

$$
d s_{11}^{2}=\eta_{\mu \nu} d x^{\mu} d x^{\nu}+d r^{2}+r^{2}\left(d \tilde{\phi}+B R d x^{11}\right)^{2}+\left(d x^{11}\right)^{2}
$$

This can be expressed as a IIA solution using the formula

$$
d s_{11}^{2}=e^{4 \phi / 3}\left(d x^{11}+R A_{\mu} d x^{\mu}\right)^{2}+e^{-2 \phi / 3} d s_{10}^{2} .
$$

One finds

$$
\begin{aligned}
d s_{10}^{2} & =\sqrt{\Lambda}\left(d r^{2}-d t^{2}+\left(d x^{1}\right)^{2}+\cdots+\left(d x^{7}\right)^{2}\right)+\frac{r^{2} d \tilde{\phi}^{2}}{\sqrt{\Lambda}} \\
A_{\tilde{\phi}} & =\frac{B r^{2}}{\Lambda}, \\
e^{\frac{4 \phi}{3}} & =\Lambda \\
\Lambda & \equiv 1+B^{2} R^{2} r^{2} .
\end{aligned}
$$

2 A similar construction involving a boost rather than a rotation leads to a time-dependent solution with nonzero electric flux. The function $\Lambda$ determining the dilaton in 2.5 becomes of the form $1+E^{2} x^{+} x^{-}$which has zeros along past and future spacelike surfaces. 
The parameter $B$ governing the size of the rotation in 2.1 is hence identified as the strength of the magnetic field at the origin, and as usual $R=\frac{g_{s}}{M_{s}}$ is the ratio of the string coupling to the string mass. Note that since radius of compactification (the coefficient of $\left.\left(d x^{11}\right)^{2}\right)$ grows like $r$, the IIA theory is strongly coupled at large $r$.

\section{2. $0 A \leftrightarrow I I A$ Duality}

In this subsection we recall an interesting duality conjectured in [7].

We first note that for $B=\frac{2}{R^{2}}=2 \frac{M_{s}^{2}}{g_{s}^{2}}$, one has a $4 \pi$ rotation in (2.1), which is equivalent to no rotation at all. Therefore IIA with this critical magnetic field is dual to the IIA vacuum! Even more provocative is the case $B=\frac{M_{s}^{2}}{g_{s}^{2}}$, for which the rotation in (2.1) has no effect on bosons but gives a minus sign for fermions. According to the conjecture of Bergmann and Gaberdiel [8], M-theory compactification on $S^{1}$ with twisted fermion boundary conditions is the $0 \mathrm{~A}$ string theory at half the string coupling. This implies that IIA theory with $B=\frac{M_{s}^{2}}{g_{s}^{2}}$ is dual to 0A string theory! More generally it was conjectured in [7] that

$$
I I A\left(B, g_{s}\right) \leftrightarrow 0 A\left(B-\frac{M_{s}^{2}}{g_{s}^{2}}, \frac{g_{s}}{2}\right) .
$$

One of the main pieces of evidence for this duality comes from further $S^{1}$ compactification to 9 dimensions, followed by a "9-11" flip [8,7]. This results in a perturbative duality relating IIA and $0 \mathrm{~A}$ on twisted circles. Indeed the partition function is exactly computable [10,11,7] and smoothly interpolates from IIA to $0 \mathrm{~A}$ as a function of $B$.

Of course ultimately the arguments in favor of this duality involve extrapolations to strong coupling which are unprotected by supersymmetry. As such they are much weaker than the arguments for supersymmetric dualities. Phase transitions could invalidate the picture. Nevertheless we feel the picture developed in [8,7] hangs together quite well and is worth pursuing.

The periodic behavior of the magnetic flux appears almost trivial from the M-theory perspective since the flux is equivalent to a rotation. However from the IIA perspective 
it is quite surprising. In this perspective a field strength is turned on, breaking both supersymmetry and Lorentz invariance. Then at a large critical value of this field strength, Lorentz invariance is restored in new dual variables. At a yet higher field strength, Lorentz invariance is again restored, together with supersymmetry. The variables of this new IIA theory are related by a complicated nonlocal transformation to those of the old IIA theory. The spectral flow relating the states of the two IIA theories mixes perturbative and nonperturbative excitations. 3

\subsection{The Fate of the Tachyon}

Recently there has been much discussion of open string tachyon condensation. In this subsection we make a speculation about the endpoint of $0 \mathrm{~A}$ (closed string) tachyon condensation.

Let us first review the F7-brane instability at strong coupling, where the M-theory picture may be used [7]. The 11-dimensional euclidean Kerr solution [12] then provides an instanton with the correct boundary conditions. It has a negative mode and so represents an instability. However the interpretation of this instability depends on whether we use the $0 \mathrm{~A}$ or IIA interpretation of the theory. In the IIA interpretation, it represents a brany analog of Schwinger pair production in which the magnetic field is damped by the creation Kaluza-Klein monopoles in a spherical shell. In the 0A interpretation one has instead a so called "bubble of nothing" [13]. It is quite challenging to understand the endpoint of the instability in the $0 \mathrm{~A}$ picture. From the IIA picture we expect the pair creation should continue until the magnetic field is completely dissipated and one reverts to the IIA vacuum.

At weak coupling, the IIA instability can be understood as the creation of zeroenergy spherical D6-branes. At small radius, a spherical D6 of course has positive energy.

3 After compactification to 9 dimensions and a 9-11 flip, the spectral flow can be followed perturbatively [10] [7]. 
However at larger radius the energy becomes zero and then negative due to the potential energy in the magnetic field.

It is natural to suppose that at weak coupling in the $0 \mathrm{~A}$ picture the instability under discussion can be identified with that of the $0 \mathrm{~A}$ tachyon. This fits with the conjecture of [8] that at a critical value of the coupling, the tachyon mass becomes positive. At this value the perturbative instability becomes nonperturbative and may be identified with the Kerr instanton instability.

This picture of closed string tachyon condensation, and its relation to a nonperturbative instability at strong coupling, has an intriguing similarity to that of open strings in the $D p-\bar{D} p$ system. The tachyon is an open string stretched between a $D p$ brane and a $\bar{D} p$-brane. Pulling the $D p-\bar{D} p$ pair sufficiently far apart raises the mass of the tachyon above zero and eliminates the perturbative instability. However it is replaced by a nonperturbative instability. This is described by an instanton which is a tube connecting the $D p-\bar{D} p$ pair, and mediates decay into a "bubble of nothingness' [14 16]. Similarly, the $0 \mathrm{~A}$ theory has a perturbative tachyon instability at weak coupling which is replaced by decay into bubbles of nothing when the tachyon gets a positive mass.

\section{4. $F^{7} \leftrightarrow$ IIA Cone Duality}

Given an 11-dimensional geometry, a circle must be chosen to obtain a 10-dimensional description. The 0A-IIA duality can be viewed as different choices of this circle. In both cases the circle lies in the torus parameterized by $x^{11}$ and the angle $\phi$ in the plane transverse to the F7-brane, but they differ by a modular transformation. More general alternate IIA descriptions of the theory, in the spirit of [17], can be obtained by a $S L(2, Z)$ transformation of the torus in (2.2)

$$
\begin{aligned}
\hat{x}^{11} & =a x^{11}+b R \tilde{\phi}, \\
\hat{\phi} & =\frac{c}{R} x^{11}+d \tilde{\phi},
\end{aligned}
$$

identified as

$$
\begin{gathered}
\hat{x}^{11} \sim \hat{x}^{11}+2 \pi n_{1} R, \\
\hat{\phi} \sim \hat{\phi}+2 \pi n_{2},
\end{gathered}
$$


where the integers $a, b, c, d$ obey $a d-b c=1$. One then finds that the flat 11-dimensional metric (2.3) becomes

$$
d s_{11}^{2}=\eta_{\mu \nu} d x^{\mu} d x^{\nu}+d r^{2}+r^{2}\left(\left(a-b B R^{2}\right) d \hat{\phi}+\left(d B R-\frac{c}{R}\right) d \hat{x}^{11}\right)^{2}+\left(d d \hat{x}^{11}-b R d \hat{\phi}\right)^{2}
$$

Consider the case that the total magnetic flux is an integer $N$. This implies

$$
B R^{2}=\frac{1}{N}
$$

We may then choose

$$
a=1, \quad b=N-1, \quad c=1, \quad d=N,
$$

which reduces (2.9) to

$$
d s_{11}^{2}=\eta_{\mu \nu} d x^{\mu} d x^{\nu}+d r^{2}+\frac{r^{2}}{N^{2}}(d \hat{\phi})^{2}+\left(N d \hat{x}^{11}-(N-1) R d \hat{\phi}\right)^{2} .
$$

This corresponds to a compactification to a locally flat ten-dimensional spacetime with a $Z_{N}$ identification about the 8 -plane $r=0$. The string coupling is

$$
g_{s}=\left(N R M_{p}\right)^{3 / 2}
$$

There is also a flat $U(1)$ connection $A_{\phi}=\frac{N-1}{N}$.

More generally one may consider the case of rational $B$ defined by

$$
B R^{2}=\frac{m}{N}
$$

for integer $m$. We may then take

$$
c=m, \quad d=N \quad N a-m b=1 .
$$

There are infinitely many $a, b$ satisfying this relation and we will choose the smallest $b$. which reduces $(2.9)$ to

$$
d s_{11}^{2}=\eta_{\mu \nu} d x^{\mu} d x^{\nu}+d r^{2}+\frac{r^{2}}{N^{2}}(d \hat{\phi})^{2}+\left(N d \hat{x}^{11}-b R d \hat{\phi}\right)^{2}
$$

This differs from (2.12) only in the flat connection.

In conclusion a F7-brane is dual to IIA on a flat cone with RR flux at the origin. This duality will have a more familiar analog in the context of the supersymmetric branes of the next section. 


\section{ALE $\leftrightarrow$ Supersymmetric F5-brane Duality}

It is interesting to consider the case where the spacetime rotation accompanying the shift of $x^{11}$ involves four (rather than two) space dimensions and lies within a $S U(2)_{L}$ subgroup of $S O(4)$. Parameterizing $R^{4}$ in terms of two complex coordinates

$$
z_{1}=x_{6}+i x_{7}=r \cos \theta e^{i(\phi+\psi)}, \quad z_{2}=x_{8}+i x_{9}=r \sin \theta e^{i(\phi-\psi)} .
$$

The identifications are given by

$$
\begin{aligned}
x^{11} & \sim x^{11}+2 \pi n_{1} R, \\
\phi & \sim \phi+2 \pi n_{2}+2 \pi n_{1} B R^{2} .
\end{aligned}
$$

Again the Killing vector used in the reduction is $q=\partial_{x_{11}}+B R \partial \phi$. Such flux tubes were described in detail in [6]. The identification then preserves half of the supersymmetries, given by spinors which are invariant under $S U(2)_{L}$.

$$
\epsilon \rightarrow e^{B R^{2}\left(\Gamma_{67}+\Gamma_{89}\right)} \epsilon
$$

The flat eleven dimensional metric $d s^{2}=-d t^{2}+\cdots\left|d z_{1}\right|^{2}+\left|d z_{2}\right|^{2}+d x_{11}^{2}$ can be expressed in the following way

$$
\begin{aligned}
d s^{2} & =-d t^{2}+d x_{1}^{2}+\cdots+d x_{5}^{2}+\Lambda\left(d x_{11}+R A_{\tilde{\phi}} d \tilde{\phi}+R A_{\psi} d \psi\right)^{2}+d r^{2}+r^{2} d \theta^{2} \\
& +\frac{r^{2}}{16 \Lambda}\left(d \tilde{\phi}^{2}+\left(1+\frac{B^{2} R^{2} r^{2} \sin ^{2} 2 \theta}{4}\right) d \psi^{2}+2\left(1+\frac{B^{2} R^{2} r^{2}(1-\cos 2 \theta)}{4}\right) d \tilde{\phi} d \psi\right)
\end{aligned}
$$

where

$$
\Lambda=e^{4 / 3 \phi}=1+\frac{B^{2} R^{2} r^{2}}{4}
$$

and

$$
A_{\tilde{\phi}}=\frac{B r^{2}}{4 \Lambda}, \quad A_{\psi}=\frac{B r^{2} \cos 2 \theta}{4 \Lambda} .
$$

This field configuration represents a flux fivebrane. The gauge fields in ten dimensions have a nonzero second Chern class

$$
\int F \wedge F=\oint_{S_{3}} A \wedge F=\frac{8 \pi^{2}}{B^{2} R^{2}}
$$


Note that the gauge field strength is not self-dual, however since the dilaton and the metric are nontrivial, they also appear in the supersymmetry transformation rules (given in the Einstein frame)

$$
\begin{gathered}
\delta \psi_{\mu}=D_{\mu} \epsilon+\frac{1}{64} e^{3 / 4 \phi}\left(\Gamma_{\mu}^{\nu \rho}-14 \delta_{\mu}^{\nu} \Gamma^{\rho}\right) \Gamma_{11} \epsilon F_{\nu \rho} \\
\delta \lambda=\frac{1}{\sqrt{2}} \partial_{\mu} \phi \Gamma^{\mu} \Gamma^{11} \epsilon+\frac{2}{16 \sqrt{2}} e^{3 / 4 \phi} \Gamma^{\mu \nu} \epsilon F_{\mu \nu}
\end{gathered}
$$

making a $1 / 2$ BPS configuration possible.

Proceeding as for the F7-brane in section 2 one finds in the case $B R^{2}=m / N$, that this supersymmetric fluxbrane is dual to the $A_{N}$ ALE geometry in IIA. There is also nonzero RR two-form flux at the origin. This can be seen by integrating the potential around one of the generators of $\pi_{1}=Z_{N}$ which gives $\frac{1}{N}$.

This construction has obvious generalization to Fp-branes of any p by simply choosing different rotations.

\section{The General Fp-brane Solution}

In this section we want to analyze the equations for $p$ fluxbranes in M-theory and string theory. A Fp-brane will have $p+1$ dimensional Poincare invariance in the 'worldvolume' and $S O(q)$ rotational invariance in the $q=D-p-1$ transverse directions.

There will be a non vanishing flux of a field strength $F_{q}$ tangent to the transverse directions. This is contrasted with the usual BPS branes which carry a charge measured by integrating the field strength over a sphere surrounding the brane. For notational convenience in this section we will use the number of transverse dimensions $q$ to label the fluxbranes, rather than $p=D-q-1$.

The nontrivial fields are the graviton, a $q$ form field strength $F_{q}$ and in the case of string theory a dilaton. The action in the Einstein frame is given by

$$
S=\frac{1}{l_{p}^{D-2}} \int d^{D} x \sqrt{g}\left(R-\frac{1}{2} \partial \phi \partial \phi-\frac{1}{2 q !} e^{a \phi} F_{q}^{2}\right) .
$$


For M-theory we have $D=11$ and set $a=0$ and $\phi=0$, the field strength $F_{4}$ has either $q=4$ for a magnetic fluxbrane or the dual $q=7$ for an electric fluxbrane.

For type II string theory we have $D=10$. There are two cases, firstly when the field strength $F_{q}$ comes from the Ramond-Ramond sector $q$ runs from $q=2$ to $q=8$ and the dilaton coupling is $a=1 / 2(5-q)$. Note that in type IIA(B) we have (RR) Fp-branes with odd(even) $p$ which is in contrast with Dp-branes which have even(odd) $p$. Secondly If the field strength (or its dual) is coming from the NS-NS antisymmetric tensor $B_{\mu \nu}$ we have a NS-F6 brane with $q=3$ and $a=-1$ and a NS F2-brane with $q=7$ and $a=+1$. In the Einstein frame the RR and NS Fp branes are simply related by $a \rightarrow-a$. In the following we will focus on the RR Fp-branes for type II.

Our ansatz for the metric is

$$
d s^{2}=e^{2 A(r)}\left(-d t^{2}+d x_{1}^{2}+\cdots+d x_{D-q-1}^{2}\right)+d r^{2}+e^{2 C(r)} d S_{q-1}^{2} .
$$

The equation of motion for the field strength $d * F=0$ can be easily solved 4

$$
F_{q}=f M_{p} e^{-(D-q) A+(q-1) C-a \phi} \epsilon_{r \alpha_{1} \cdots \alpha_{q-1}}
$$

The dimensionless constant $f$ measures the field strength at the origin. All functions in the ansatz only depend on the radial coordinate $r$, hence the equations of motion can be derived from a one dimensional Lagrangian $L=T-V$ where the kinetic term is given by $T=e^{(D-q) A+(q-1) C}\left(-\frac{1}{2} \phi^{\prime} \phi^{\prime}+(D-q)(D-q-1) A^{\prime} A^{\prime}+(q-1)(q-2) C^{\prime} C^{\prime}+2(q-1)(D-q) A^{\prime} C^{\prime}\right)$.

The potential term comes from the field strength and curvature terms

$$
V=-e^{(D-q) A+(q-1) C}\left((q-1)(q-2) e^{-2 C}+\frac{f^{2} M_{p}^{2}}{2} e^{-2(D-q) A-a \phi}\right) .
$$

Together with a 'zero energy constraint', coming from the $R_{r r}$ component of Einstein's equation.

$$
E=T+V=0
$$

4 In the case of the IIB F4-brane, one has to impose the self duality of the five form field strength, $F_{5}=f M_{p}\left(e^{-5 A+4 C} \epsilon_{r \alpha_{1} \cdots \alpha_{4}}+\epsilon_{t x_{1} \cdots x_{4}}\right)$ 


\subsection{The Attractor Solutions}

Before entering into a detailed discussion of the solutions we highlight some salient features. The M-theory solutions have the asymptotic form (in appropriate coordinates) at large radius

$$
d s^{2} \sim r^{m}\left(-d t^{2}+\left(d x_{1}\right)^{2}+\cdots+\left(d x_{p}\right)^{2}\right)+d r^{2}+n r^{2} d \Omega_{q-1}^{2}
$$

where $p+q=10$. The field strength behaves in these coordinates as

$$
F_{q} \sim s r^{q-2} \epsilon_{q}
$$

$s, m$ and $n$ are positive constants which depend only on $q$, and $\epsilon_{q}$ is the transverse volume. In particular, although the field strength and metric are not flat, the asymptotic form of the solution has no memory of the field strength at the origin.

Corrections to (4.7) and (4.8) are a power series in $\frac{1}{r f M_{p}}$, where $f$ is the dimensionless field strength at the origin. The larger $f$ is, the closer we can get to the origin before corrections become important. Very near the origin space is flat. The solution may be viewed as a bowl with a flat bottom. When the field strength $f$ is small, one must go far from the origin before spacetime curvature becomes important, and the flat bottom is large.

In string theory cases there is also a dilaton, and a symmetry under shifting the dilaton and rescaling $F$. This leads to one extra parameter in both the asymptotic and exact solutions, which can be taken to be the constant value of the dilaton at the origin. Otherwise the situation is similar. There are attractor solutions of the form (4.7)-(4.8) to which all solutions tend. In M-theory there is a one parameter family of solutions for each q which tend to a unique attractor, while in string theory there is a two parameter family of solutions which tend to a one parameter family of attractors labeled by the string coupling. 


\subsection{The M-Theory F6-brane}

There are two fluxbranes in M-theory: the 'magnetic' Fp-brane with $q=4$ where $F_{4}$ is non vanishing, and the 'electric' F3-brane with $q=7$ case where the dual field strength $F_{7}$ is non vanishing. In this subsection we consider the F6-brane. Setting $D=11$ and $q=4$ in the Lagrangian one arrives at the following equations of motion

$$
\begin{aligned}
A^{\prime \prime}+7 A^{\prime} A^{\prime}+3 A^{\prime} C^{\prime}-\frac{f^{2} M_{p}^{2}}{6} e^{-14 A} & =0, \\
C^{\prime \prime}+3 C^{\prime} C^{\prime}+7 A^{\prime} C^{\prime}+\frac{f^{2} M_{p}^{2}}{3} e^{-14 A}-2 e^{-2 C} & =0 .
\end{aligned}
$$

The field strength is then given by

$$
F_{r \theta \phi \psi}=f M_{p} e^{-7 A+3 C}
$$

Where $\theta, \phi, \psi$ denote angular coordinates on the transverse three sphere. The zero energy constraint (4.6) becomes

$$
E=7 A^{\prime} A^{\prime}+7 A^{\prime} C^{\prime}+C^{\prime} C^{\prime}-\frac{f^{2} M_{p}^{2}}{12} e^{-14 A}-e^{-2 C}=0 .
$$

The boundary conditions at $r=0$ are determined by the fact that at the center of the fluxbrane the metric becomes flat. This implies that

$$
C(r)=\ln r+B(r),
$$

Where both $A$ and $B$ go to constants as $r \rightarrow 0$. The behavior of the solution near $r=0$ can then be determined by a power series expansion, which depends on $a_{0}=\left.A\right|_{r=0}$.

$$
\begin{aligned}
& A(r)=a_{0}+\frac{1}{48} e^{-14 a_{0}}\left(r f M_{p}\right)^{2}-\frac{1}{2} \frac{5}{1152} e^{-28 a_{0}}\left(r f M_{p}\right)^{4}+\mathcal{O}\left(r^{6}\right), \\
& B(r)=-\frac{5}{144} e^{-14 a_{0}}\left(r f M_{p}\right)^{2}+\frac{1}{2} \frac{437}{51840} e^{-28 a_{0}}\left(r f M_{p}\right)^{4}+\mathcal{O}\left(r^{6}\right) .
\end{aligned}
$$

Note that only even powers of $r$ appear in the power series. Without loss of generality we can fix $a_{0}=0$. 
We have been unable to find an analytic solution of the equations (4.9) with the regular boundary conditions given above. It seems impossible to decouple the equations because of the potential term $e^{-2 C}$ in (4.9) which is present when $q \neq 2$, i.e. for all cases but the Melvin fluxtube. However we have been able to find an exact solution which is singular at $r=0$ :

$$
\begin{aligned}
& A(r)=\frac{1}{7} \ln \left(r f M_{p}\right)-\frac{1}{14} \ln (18 / 7), \\
& C(r)=\ln (r)-\frac{1}{2} \ln (27 / 14) .
\end{aligned}
$$

From (4.10) it follows that the behavior of the field strength is given by

$$
F_{r \alpha_{1} \alpha_{2} \alpha_{3}}=\left(\frac{2^{4} 7^{2}}{3^{7}}\right)^{1 / 2} r^{2} .
$$

Fluxbrane solutions which are regular at the origin exist and can be studied numerically. The result is that the solutions approach (4.14) asymptotically as $r \rightarrow \infty$. In addition it can be shown that there is a three parameter family of linearized perturbations around the solution (4.14), which all decay at least as fast as $1 / r$ when $r \rightarrow \infty$, so that (4.14)-(4.15) is an attractor solution.

The flux $\Phi=\oint_{S^{3}} A_{3}$ inside a sphere of radius $r$ in the transverse space grows like $\Phi \sim r^{3}$ as $r \rightarrow \infty$. This means that unlike for the case of the Melvin fluxtube, the flux of F6-brane spreads out over the transverse space.

To compare with the BPS branes it is convenient to make a change of the radial coordinate to bring the metric into 'isotropic' form. Up to an overall numerical factor one finds

$$
d s^{2} \sim\left(\tilde{r} f M_{p}\right)^{7 / 3}\left(\frac{-d t^{2}+d x_{1}^{2}+\cdots d x_{5}^{2}+d \tilde{r}^{2}}{\tilde{r}^{2}}+\frac{8}{21} d \Omega_{S_{3}}^{2}\right) .
$$

\subsection{The M-Theory F3-brane}

The F3-brane can be obtained by setting $q=7, D=11$ and $a=0$ in (4.1). The equations of motion are

$$
\begin{aligned}
A^{\prime \prime}+4 A^{\prime} A^{\prime}+6 A^{\prime} C^{\prime}-\frac{f^{2} M_{p}^{2}}{3} e^{-8 A} & =0, \\
C^{\prime \prime}+6 C^{\prime} C^{\prime}+4 A^{\prime} C^{\prime}+\frac{f^{2} M_{p}^{2}}{6} e^{-8 A}-5 e^{-2 C} & =0 .
\end{aligned}
$$


The zero energy constraint becomes

$$
E=A^{\prime} A^{\prime}+\frac{5}{2} C^{\prime} C^{\prime}+4 A^{\prime} C^{\prime}-\frac{f^{2} M_{p}^{2}}{24} e^{-8 A}-\frac{5}{2} e^{-2 C}=0 .
$$

The field strength is given by

$$
F_{r \alpha_{1} \cdots \alpha_{6}}=f M_{p} e^{-4 A+6 C} .
$$

Where $\alpha_{1}, \cdots, \alpha_{6}$ denote the coordinates on the transverse six sphere. Repeating the analysis for the F6-brane given in the previous section one finds that the behavior of the fluxbrane solution near $r \rightarrow 0$ is

$$
\begin{aligned}
& A(x)=a_{0}+\frac{1}{42} e^{-8 a_{0}}\left(r f M_{p}\right)^{2}-\frac{1}{2} \frac{59}{15876} e^{-16 a_{0}}\left(r f M_{p}\right)^{4}+\mathcal{O}\left(r^{6}\right), \\
& B(x)=-\frac{5}{504} e^{-8 a_{0}}\left(r f M_{p}\right)^{2}+\frac{1}{2} \frac{1159}{635040} e^{-16 a_{0}}\left(r f M_{p}\right)^{4}+\mathcal{O}\left(r^{6}\right) .
\end{aligned}
$$

Using numerical and perturbation methods as in section 4.1 one can find good evidence that the regular fluxbrane solution will behave asymptotically as $r \rightarrow \infty$ like

$$
\begin{aligned}
& A(r)=\frac{1}{4} \ln \left(r f M_{p}\right)-\frac{1}{8} \ln (9 / 2)+\mathcal{O}(1 / r), \\
& C(r)=\ln r-\frac{1}{2} \ln (27 / 20)+\mathcal{O}(1 / r) .
\end{aligned}
$$

Using (4.3) it is easy to see that the flux will grow like $\Phi=r^{6}$ as $r \rightarrow \infty$. In an isotropic coordinate system the asymptotic form of metric will be

$$
d s^{2} \sim\left(\tilde{r} f M_{p}\right)^{8 / 3}\left(\frac{-d t^{2}+d x_{1}^{2}+\cdots d x_{3}^{2}+d \tilde{r}^{2}}{\tilde{r}^{2}}+\frac{5}{12} d \Omega_{S_{6}}^{2}\right) .
$$

\subsection{Type II fluxbranes}

In type II case there is a dilaton in the action (4.1). Setting $D=10$ and the dilaton coupling $a=1 / 2(5-q)$ in the Lagrangian the equations of motion in the Einstein frame become

$$
\begin{aligned}
\phi^{\prime \prime}+\phi^{\prime}\left((10-q) A^{\prime}+(q-1) C^{\prime}\right)-\frac{1}{4}(5-q) f^{2} M_{s}^{2} e^{-2(10-q) A-\frac{1}{2}(5-q) \phi} & =0, \\
A^{\prime \prime}+A^{\prime}\left((10-q) A^{\prime}+(q-1) C^{\prime}\right)-\frac{q-1}{16} f^{2} M_{s}^{2} e^{-2(10-q) A-\frac{1}{2}(5-q) \phi} & =0, \\
C^{\prime \prime}+C^{\prime}\left((10-q) A^{\prime}+(q-1) C^{\prime}\right)-(q-2) e^{-2 C}+\frac{9-q}{16} f^{2} M_{s}^{2} e^{-2(10-q) A-\frac{1}{2}(5-q) \phi} & =0 .
\end{aligned}
$$


The zero energy constraint becomes

$$
\begin{aligned}
E & =-\frac{1}{2} \phi^{\prime} \phi^{\prime}+(10-q)(9-q) A^{\prime} A^{\prime}+(q-1)(q-2) C^{\prime} C^{\prime}+2(q-1)(10-q) A^{\prime} C^{\prime} \\
& -(q-1)(q-2) e^{-2 C}-\frac{1}{2} f^{2} M_{s}^{2} e^{-2(10-q) A-\frac{1}{2}(5-q) \phi}=0 .
\end{aligned}
$$

There is a simple integral of motion which sets

$$
\phi=4 \frac{5-q}{q-1} A
$$

Note that when $q=5$, i.e. for the type IIB F4-brane, the dilaton is a constant and decouples. Eliminating $\phi$ from the equations (4.23) reduces the system to

$$
\begin{array}{r}
A^{\prime \prime}+A^{\prime}\left((10-q) A^{\prime}+(q-1) C^{\prime}\right)-\frac{q-1}{16}\left(f M_{s}\right)^{2} e^{-2 \frac{15+q}{q-1} A}=0, \\
C^{\prime \prime}+C^{\prime}\left((10-q) A^{\prime}+(q-1) C^{\prime}\right)-(q-2) e^{-2 C}+\frac{9-q}{16}\left(f M_{s}\right)^{2} e^{-2 \frac{15+q}{q-1} A}=0,
\end{array}
$$

Hence the complexity of the equations for the type II fluxbranes is the same as the one for M-theory fluxbranes. Note that the Melvin fluxtube is the type IIA F7-brane which has $q=2$. In this case the $e^{-2 C}$ term disappear 4.26 ) and the equations can be solved exactly, yielding the solution (2.5).

Making the same assumptions of regularity at $r=0$ as for the M-fluxbranes implies that the solution behaves near the origin as

$$
\begin{aligned}
& A(r)=a_{0}+\frac{q-1}{32 q} e^{-2 \frac{15+q}{q-1} a_{0}}\left(r f M_{s}\right)^{2}+\mathcal{O}\left(r^{4}\right), \\
& B(r)=\frac{5-10 q+q^{2}}{48 q(q-1)} e^{-2 \frac{15+q}{q-1} a_{0}}\left(r f M_{s}\right)^{2}+\mathcal{O}\left(r^{4}\right) .
\end{aligned}
$$

Numerical analysis of the differential equations (4.26) shows that in the limit $r \rightarrow \infty$ the solution behaves like

$$
\begin{aligned}
& A(r)=\frac{q-1}{15+q} \ln \left(r f M_{s}\right)-\frac{q-1}{2(15+q)} \ln \left(\frac{128(3 q-5)}{(15+q)^{2}}\right)+\mathcal{O}(1 / r), \\
& C(r)=\ln (r)-\frac{1}{2} \ln \left(\frac{192(3 q-5)}{(q-2)(15+q)^{2}}\right)+\mathcal{O}(1 / r) .
\end{aligned}
$$

The asymptotic behavior of the dilaton is then

$$
e^{\phi} \sim\left(r f M_{s}\right)^{4 \frac{(5-q)}{15+q}} .
$$


This implies that the coupling blows up for $q<5$, i.e. for $p>4$ Fp-branes. As $r \rightarrow \infty$ the form of the type II $q$ fluxbranes is given by

$$
d s^{2} \sim\left(r f M_{s}\right)^{\frac{q-1}{15+q}}\left(\frac{128(3 q-5)}{(15+q)^{2}}\right)^{-\frac{q-1}{15+q}}\left(-d t^{2}+d x_{1}^{2}+\cdots+d x_{9-q}^{2}\right)+d r^{2}+\frac{(q-2)(15+q)^{2}}{192(3 q-5)} r^{2} d S_{q-1}^{2} .
$$

In the new coordinates the asymptotic metric in the Einstein frame takes the form

$$
d s^{2} \sim\left(\tilde{r} f M_{s}\right)^{\frac{15+q}{8}}\left(\frac{-d t^{2}+d x_{1}^{2}+\cdots+d x_{9-q}^{2}+d \tilde{r}^{2}}{\tilde{r}^{2}}+\frac{4}{3} \frac{(q-2)}{(3 q-5)} d S_{q-1}^{2}\right),
$$

where we have dropped an overall numerical factor. The asymptotic behavior of the dilaton is given by

$$
e^{\phi} \sim\left(\tilde{r} f M_{s}\right)^{\frac{5-q}{4}}
$$

To compare this with the near horizon geometry of $p$ branes, we have to transform the metric to the string frame, i.e. multiply (4.31) by $e^{\phi / 2}$. Here we find a surprise: The conformal factor of the metric does not depend on $q$ :

$$
d s^{2} \sim\left(\tilde{r} f M_{s}\right)^{5 / 2}\left(\frac{-d t^{2}+d x_{1}^{2}+\cdots+d x_{9-q}^{2}+d \tilde{r}^{2}}{\tilde{r}^{2}}+\frac{4}{3} \frac{(q-2)}{(3 q-5)} d S_{q-1}^{2}\right)
$$

and the dilaton behavior is given by (4.32).

\section{Critical D(p-1)-branes in Fp-branes}

In this section we consider D-branes in a fluxbrane. In particular a $\mathrm{D}(\mathrm{p}-1)$-brane couples to the flux in a Fp-brane. We will describe a new scaling limit of $\mathrm{D}(\mathrm{p}-1)$ branes in Fp-branes.

\subsection{The Critical M2-brane in a F3-brane}

We begin with the simplest case which is the M2-brane. The bosonic part of the action is

$$
S_{2}=-M_{p}^{3} \int d^{3} \sigma \sqrt{-\operatorname{det}\left(g_{\mu \nu} \partial_{i} X^{\mu} \partial_{j} X^{\nu}\right)}+M_{p}^{3} \int \frac{1}{3 !} C_{\mu \nu \rho} d X^{\mu} d X^{\nu} d X^{\rho}
$$


We consider the background

$$
g_{\mu \nu}=\eta_{\mu \nu}, \quad C_{0 \nu \rho}=f M_{p} \epsilon_{0 \nu \rho \lambda} X^{\lambda}
$$

where $f$ is dimensionless and $\epsilon$ is the volume element of a flat $(3+1)$-dimensional subspace. (5.2) is a flat metric with a constant field strength $F_{4}=d C=f M_{p} \epsilon$. Of course such a field strength acts as a source for metric curvature, so (5.2) is valid only in a small neighborhood of the origin. We will correct for this later when we consider fluctuations of the membrane.

Now consider a spherical M2-brane of radius $R$ oriented tangent to the flux $F_{4}=d C$. (5.2). The energy of such a brane is up to an overall numerical factor

$$
E(R)=M_{p}^{3} R^{2}-f M_{p}^{4} R^{3}
$$

The first, positive, term is just the total tension of the brane. The second arises from the energy in the $F$ field. It is negative because the field strength is smaller in the interior of the spherical M2-brane (for the chosen brane orientation). The energy is stationary at

$$
R_{0}=\frac{2}{3} \frac{1}{f M_{p}}
$$

This corresponds to a static but unstable M2-brane configuration.

This configuration has a simple analog. Consider letting go of an electron-positron pair in an electric field. If they are initially far apart, their mutual attraction is negligible and they will be accelerated further apart by the electric force. If they are initially nearby, they will attract and annihilate. In between these two extremes is a critical radius at which they can remain in unstable equilibrium. This configuration is the analog of the unstable M2-brane. Note that both the M2-brane and the electron-positron pair have no net charge.

A similar solution exists in euclidean space

$$
g_{\mu \nu}=\delta_{\mu \nu}, \quad C_{\mu \nu \rho}=f M_{p} \epsilon_{\mu \nu \rho \lambda} X^{\lambda}
$$


with a $S^{3}$ (rather than $S^{2} \times R$ ) M2-brane. The critical radius for this solution is

$$
R_{0}=\frac{3}{4} \frac{1}{f M_{p}}
$$

This is an instanton which describes decay of a constant $F$ field through spherical brane creation [6]. The branes are created with zero energy according to (5.3). They subsequently expand out to infinity, leaving a dampened $F$ field in their interior. The brane worldvolume is the deSitter space $d S_{3}$. This is the brane analog of Schwinger pair production [6].

Now we wish to consider a scaling limit in which we take the Planck mass to infinity while holding the critical radius (5.4) (or equivalently (5.6)) fixed:

$$
M_{p} \rightarrow \infty, \quad f \rightarrow 0 \quad R_{0}=\frac{2}{3} \frac{1}{f M_{p}} \quad \text { fixed }
$$

Note that this requires a weak dimensionless field strength. In order to compute the membrane field theory in this limit, the approximate solution (5.5) is inadequate. We must use instead (4.20). The M2-brane action (5.1) near unstable equilibrium for topology $S^{2} \times R$ can be written as

$$
\begin{aligned}
& S_{2}=-M_{p}^{3} \int d^{3} \sigma\left(\sqrt{-\operatorname{det}\left(\partial_{i} \rho \partial_{j} \rho+e^{2 C(\rho)} \eta_{\alpha \beta} \partial_{i} X^{\alpha} \partial_{j} X^{\beta}+e^{2 A(\rho)}\left(\partial_{i} R \partial_{j} R+\frac{R^{2}}{R_{0}^{2}} h_{i j}\right)\right.}\right) \\
& \left.\quad+\sqrt{-h} f M_{p} \frac{R^{3}}{R_{0}^{2}}\right) .
\end{aligned}
$$

In this expression $h_{i j}$ is the radius $R_{0}$ (as given in (5.6)) metric on $S^{2} \times R, X^{m}$ for $m, n=4, \cdots, 10$ are fields in the 7 directions orthogonal to the worldvolume directions of the fluxbrane, $\rho^{2} \equiv \delta_{m n} X^{m} X^{n}, R$ is a field tangent to the fluxbrane but orthogonal to the spherical brane, $A$ and $B$ are given in $(4.20)$ with $a_{0}=0$ and the three longitudinal fields in (5.1) have been eliminated by gauge fixing. Next we define fluctuations about the static solution by

$$
R=R_{0}+\frac{U}{M_{p}^{3 / 2}}, \quad X^{m}=\frac{U^{m}}{M_{p}^{3 / 2}}
$$


Near the scaling limit $(5.7)$ the action then becomes

$$
S_{2}=-\int d^{3} \sigma \sqrt{-h}\left(\frac{1}{3} M_{p}^{3}+\delta_{m n} \nabla^{j} U^{m} \nabla_{j} U^{n}+\frac{2}{7 \dot{9}} \frac{\delta_{m n} U^{m} U^{n}}{R_{0}^{2}}+\nabla^{j} U \nabla_{j} U-\frac{U^{2}}{R_{0}^{2}}\right)+\mathcal{O}\left(\frac{1}{M_{p}^{2}}\right) .
$$

Note that the mass $m_{U}^{2}=-\frac{1}{R_{0}^{2}}$ of the tachyon $U$ is finite for $M_{p} \rightarrow \infty$.

There is also a critical theory for a $d S_{3}$ brane with a $S O(3,1)$ symmetry and, in euclidean space, with $S O(4)$ symmetry. In the latter case one finds the euclidean version of (5.10) but with $R_{0}$ given by (5.6) and where the mass of the tachyonic excitation is $m_{U}^{2}=-3 /\left(2 R_{0}^{2}\right)$.

Of course the theory (5.10) is free for a single M2-brane. The theory for $N$ M2-branes is strongly interacting at low energies but poorly understood, even for a flat worldvolume. We will be more explicit about the interacting theory for some D-brane cases.

\subsection{The Critical M5-brane in a F6-brane}

Let us now consider the analogous story for the M5-brane. The action for the scalar fields is

$$
S_{5}=-M_{p}^{6} \int d^{6} \sigma \sqrt{-\operatorname{det}\left(g_{\mu \nu} \partial_{i} X^{\mu} \partial_{j} X^{\nu}\right)}+M_{p}^{6} \int \frac{1}{6 !} \tilde{C}_{\mu_{1} \cdots \mu_{6}} d X^{\mu_{1}} \cdots d X^{\mu_{6}}
$$

where (when the Chern-Simons eleven-form can be ignored) $\tilde{C}$ is defined by $* F=d \tilde{C}$. We consider the background

$$
g_{\mu \nu}=\eta_{\mu \nu}, \quad \tilde{C}_{0 \mu_{2} \cdots \mu_{6}}=f M_{p} \epsilon_{0 \mu_{2} \cdots \mu_{7}} X^{\mu_{7}}
$$

where $f$ is dimensionless and $\epsilon$ here is the volume element of a flat $(6+1)$-dimensional subspace. This gives a constant field strength $F=* d \tilde{C}=f M_{p} * \epsilon$ which differs from the M2-brane case in that it is purely spatial and has no time components. It corresponds to the field strength of a F6-brane.

Now consider a spherical M5 of radius $R$ in the background (5.12). The energy of such a brane is

$$
E(R)=M_{p}^{6} R^{5}-f M_{p}^{7} R^{6} .
$$


The energy is stationary at

$$
R_{0}=\frac{5}{6} \frac{1}{f M_{p}} .
$$

This corresponds to a static but unstable M5 configuration. Holding this radius fixed with $M_{p} \rightarrow \infty$ yields the critical M5-brane theory. Defining

$$
R=R_{0}+\frac{U}{M_{p}^{3}}, \quad X^{m}=\frac{U^{m}}{M_{p}^{3}} .
$$

the action scales

$$
S_{5}=-\int d^{6} \sigma \sqrt{-h}\left(\frac{1}{6} M_{p}^{6}+\delta_{m n} \nabla^{j} U^{m} \nabla_{j} U^{n}+\frac{5^{2}}{2^{5} 3^{2}} \frac{\delta_{m n} U^{m} U^{n}}{R_{0}^{2}}+\nabla^{j} U \nabla_{j} U-\frac{5}{2} \frac{U^{2}}{R_{0}^{2}}\right)+\mathcal{O}\left(\frac{1}{M_{p}^{2}}\right) .
$$

In this expression $m, n$ run over 4 transverse dimensions.

In the euclidean version, one has a critical M5-brane solution with topology $S^{6}$ and radius

$$
R_{0}=\frac{6}{7} \frac{1}{f M_{p}} .
$$

In the scaling limit the action has the same structure as (5.16) where the mass of the tachyonic mode is $m_{R}^{2}=-3 / R_{0}^{2}$ and the mass of the transverse fluctuations is given by $m_{U^{n}}^{2}=9 /\left(2 R_{0}^{2}\right)$.

In addition there are fermions and a self dual antisymmetric tensor field $H=* H$ on the worldvolume. This couples to the scalar fields through the term $\int C \wedge H$ with $C$ as in (5.5). At distances short compared to $R_{0}$ one recovers the $S O(7,1) \times S O(5)$ superconformally invariant $(0,2)$ theory.

\subsection{The Critical Dp-brane in a $F(p+1)$-brane}

The case of D-branes is only slightly more complicated. Consider the DBI-WZW action for a Dp-brane

$$
\begin{aligned}
S_{p} & =-\frac{M_{s}^{p+1}}{g_{s}} \int d^{p+1} \sigma e^{-\phi} \sqrt{-\operatorname{det}\left(g_{\mu \nu} \partial_{i} X^{\mu} \partial_{j} X^{\nu}+\frac{2 \pi}{M_{s}^{2}} F_{i j}\right)} \\
& +M_{s}^{p+1} \int \frac{1}{(p+1) !} C_{\mu_{1} \cdots \mu_{p+1}} d X^{\mu_{1}} \cdots d X^{\mu_{p+1}},
\end{aligned}
$$


in the $\mathrm{F}(\mathrm{p}+1)$-brane background of section 4 . Near the origin we have the background

$$
g_{\mu \nu}=\eta_{\mu \nu}, \quad C_{0 \mu_{2} \cdots \mu_{p+1}}=f M_{s} \epsilon_{0 \mu_{2} \cdots \mu_{p+2}} X^{\mu_{p+2}},
$$

where $f$ is dimensionless and $\epsilon$ is the volume element of a flat $(\mathrm{p}+2)$-dimensional subspace. There is an unstable $S^{p} \times R$ brane solution at the critical radius

$$
R_{0}=\frac{p}{p+1} \frac{1}{f g_{s} M_{s}}=\frac{p}{p+1} \frac{1}{f g_{Y M}^{2} M_{s}^{p-2}},
$$

where $g_{Y M}^{2}=g_{s} M_{s}^{3-p}$ is the D-brane gauge coupling. Now we wish to take a scaling limit

$$
M_{s} \rightarrow \infty, \quad R_{0}, \quad g_{Y M}^{2} \text { fixed. }
$$

The dimensionless field strength $f$ behaves as

$$
f=\frac{p}{p+1} \frac{M_{s}^{2-p}}{R_{0} g_{Y M}^{2}},
$$

which goes to zero or infinity depending on the value of $p$. As before we introduce scaling variables for the transverse fluctuations

$$
U^{m}=M_{s}^{2} X^{m}, \quad m=1, \cdots, 8-p, \quad U=M_{s}^{2} R,
$$

while the D-brane gauge field is not scaled. The power of $M_{s}^{2}$ insures that the kinetic terms remain finite as $M_{s} \rightarrow \infty$, as well as the (tachyonic) mass term for $U$,

$$
m_{U}^{2}=-\frac{p}{2} \frac{1}{g_{Y M}^{2} R_{0}^{2}}
$$

However the mass $m_{T}$ for the fields $U^{m}$ transverse to the Fp-brane arise from quadratic corrections to the metric and dilaton given in (4.27) where the metric has to be transformed to the string frame. These are a power series in $f M_{s} X^{m}$ which scales as

$$
f M_{s} X^{m} \sim \frac{M_{s}^{1-p} U^{m}}{R_{0} g_{Y M}^{2}} .
$$


Since the entire action is multiplied by $\frac{M_{s}^{4}}{g_{Y M}^{2}}$, the mass goes as

$$
m_{U^{n}}^{2}=\frac{5 p^{2}}{8(9-p)(p+1)^{2}} \frac{M_{s}^{6-2 p}}{R_{0}^{2} g_{Y M}^{6}} .
$$

Hence for $p>3$, the mass scales to zero. For $p=3$ it is finite but higher order corrections scale to zero. If $p<3, m_{T}^{2}$ scales to infinity and the fields $U^{m}$ are frozen.

The resulting critical theory is similar to (5.10) but of course also has $U(N)$ gauge fields (for the case of $N$ coincident branes). In the case of $p=3$ at distances short compared to $R_{0}$, one has the superconformal $\mathcal{N}=4$ Yang-Mills. At scales of order $R_{0}$ the superconformal symmetry is softly broken by for example a tachyonic mass term for one of the six scalars.

\section{A Holographic Dual?}

It is natural to conjecture, in the spirit of $\mathrm{AdS} / \mathrm{CFT}$ [18], that the F-branes in string theory and $M$-theory are the large $N$ duals of field theories without gravity. This would be of special interest because the duals should be nonsupersymmetric field theories. Several attempts to understand nonsupersymmetric holographic dualities 19-23 have proceeded by looking for spacetime solutions corresponding to nonsupersymmetric brane configurations. These have in general turned out to be singular at the origin, which has hampered progress. Here we are taking the opposite tack: we are beginning form a smooth spacetime solution and trying to find a field theory dual. Of course any such duality conjecture will be difficult to verify because there is no supersymmetry and the solutions are unstable. In this section we will mention several possibilities.

Recalling the type II solutions of subsection 4.3, at large radius the Fp-brane dilaton and metric are

$$
\begin{gathered}
e^{\phi}=\left(\tilde{r} f M_{s}\right)^{\frac{p-4}{4}} \\
d s^{2}=\left(\tilde{r} f M_{s}\right)^{5 / 2}\left(\frac{-d t^{2}+d x_{1}^{2}+\cdots+d x_{p}^{2}+d \tilde{r}^{2}}{\tilde{r}^{2}}+\frac{4}{3} \frac{(7-p)}{(22-3 p)} d S_{8-p}^{2}\right) .
\end{gathered}
$$


The solution is the same in form as the near horizon D-brane solutions, but the coefficients differ. The fact that the blueshift factor $g_{t t} \rightarrow \infty$ as $r \rightarrow \infty$ suggests that $r=\infty$ corresponds to the UV.

What could the dual field theory be? One possibility that comes to mind is the field theory of N non-BPS D-branes. Like the Fp-branes, these exist only for odd (even) p in type IIA (IIB) string theory. However there are a number of question to which we have no answer, including: What happens to the tachyon on the non-BPS brane? What is $\mathrm{N}$ dual to? What is the origin of the RR flux? Why does the dilaton blow up only for $p>4$, since field theories tend to be well behaved in the UV only for $p<4$ ?

Another possibility, which addresses some of these questions but raises others, is the critical D-brane bubbles of the previous section. The behavior of the dilaton suggests that $\mathrm{p}$ is the dimension of the dual field theory plus one. This matches with the fact that the critical brane has dimension one less than the Fp-brane in which it lies. It also is naturally associated with RR flux. However a problem with this idea is that the field theory on the critical D3-brane in the F4-brane is N=4 Yang-Mills in the UV. Hence we would expect the holographic dual to be asymptotic to $A d S_{5} \times S^{5}$. This does not seem to be the case.

A further problem with this proposal is that these bubbles are not the conformal boundaries of the fluxbranes. In the $A d S^{5} \times S^{5}$ example, the branes live on the $S^{3} \times R$ conformal boundary of the spacetime. (Of course it is not clear which features of $A d S$ holography should carry over.) The conformal boundary of the metric (6.2) at $r=\infty$ is an $8-p$ sphere. This suggests the possibility of a dual field theory on $S^{8-p}$, but we have no concrete proposal for what that might be.

It is also of interest to consider a dual DLCQ matrix description of the F7-brane. Interesting progress in the direction has recently been made in [24].

In conclusion holographic duality for Fp-branes remains an interesting and open problem. 


\section{Flux Periodicity}

The IIA F7-brane has a self-duality under shifts of $2 \pi$ in the magnitude of the twofrom field strength at the origin. This follows from its presentation as a twisted M-theory compactification. Although there is no simple M-argument, it is natural to expect that the other fluxbranes also have a periodic character. Indirect evidence for this follows from T-duality. A longitudinal T-duality of the IIA F7-brane gives the IIB F6-brane, smeared along the direction of the T-duality. This implies that the smeared - and possibly also the unsmeared - F6 is periodic (and is dual to $0 \mathrm{~B}$ at a critical field strength). Further T-duality similarly suggests that all the fluxbranes may be periodic.

Further indirect evidence for periodicity can be found from examination of the flux decay rate. The IIA F7-brane decays by production of spherical D6-branes. In the Mtheory picture these are Kaluza-Klein monopoles. Naively one expects the decay rate to grow with the field strength. However the instanton action goes to infinity at the critical field $B=\frac{2}{R^{2}}$ implying there is no decay. This is consistent with the dual reinterpretation of this critical field as the flat IIA vacuum.

A similar phenomenon occurs in original non-periodic four-dimensional Melvin universe. This is a solution of $N=2$ supergravity with no vector multiplets, which can embedded in string theory for example by IIB compactification on a rigid Calabi-Yau. This Melvin universe decays by pair production of charged black holes rather than KaluzaKlein monopoles. The instanton action for production of a pair of black holes with integer magnetic charges $\pm q$ in a magnetic field $B$ is 25,26

$$
S=4 \pi q^{2} \frac{\left(1-B q^{2}\right)}{1-(1-B q)^{4}} .
$$

The action goes to infinity, and the decay rate to zero, when

$$
B=\frac{2}{q}
$$

The reason for this is that the black holes are pair created at a separation of order $\frac{1}{B}$. When $B$ gets to large, the black hole horizons touch and the pair cannot be pulled apart. 
At the critical field $B=2$ all pair production ceases. This suggests that we have reached a new supersymmetric vacuum - possibly just a dual representation of the $B=0$ vacuum.

\section{Note added:}

After this work was completed, [27] appeared in which the singular fluxbrane solutions presented in section 4 were found independently.

\section{Acknowledgements}

We are grateful to Oren Bergman, Miguel Costa, Rajesh Gopakumar, Gary Horowitz, Shiraz Minwalla, Lubos Motl, Eva Silverstein and Cumrun Vafa for useful discussions. The work of M.G. is supported in part by the David and Lucile Packard Foundation and that of A.S. by DOE grant DE-FG02-91ER40654. 


\section{References}

[1] M. A. Melvin, "Pure Magnetic and Electric Geons", Phys. Lett. 8 (1964) 65.

[2] G. W. Gibbons, "Quantized Flux Tubes In Einstein-Maxwell Theory And Noncompact Internal Spaces", Print-86-0411 (CAMBRIDGE) Presented at 22nd Karpacz Winter School of Theoretical Physics: Fields and Geometry, Karpacz, Poland, Feb 17 - Mar 1,1986 .

[3] G. W. Gibbons and K. Maeda, "Black Holes And Membranes In Higher Dimensional Theories With Dilaton Fields", Nucl. Phys. B298 (1988) 741.

[4] F. Dowker, J. P. Gauntlett, S. B. Giddings and G. T. Horowitz, "On pair creation of extremal black holes and Kaluza-Klein monopoles", Phys. Rev. D50 (1994) 2662 hep-th/9312172.

[5] F. Dowker, J. P. Gauntlett, G. W. Gibbons and G. T. Horowitz, "The Decay of magnetic fields in Kaluza-Klein theory," Phys. Rev. D 52 (1995) 6929 [hep-th/9507143].

[6] F. Dowker, J. P. Gauntlett, G. W. Gibbons and G. T. Horowitz, "Nucleation of PBranes and Fundamental Strings", Phys. Rev. D53 (1996) 7115 [hep-th/9512154].

[7] M. Costa and M. Gutperle, "The Kaluza-Klein Melvin Solution in M-theory", hepth/0012072].

[8] O. Bergman and M. R. Gaberdiel, "Dualities of type 0 strings", JHEP 9907 (1999) 022 hhep-th/9906055].

[9] C. Chen, D. V. Gal'tsov and S. A. Sharakin, "Intersecting M-fluxbranes," Grav. Cosmol. 5 (1999) 45 hep-th/9908132.

[10] J. G. Russo and A. A. Tseytlin, "Magnetic flux tube models in superstring theory", Nucl. Phys. B461 (1996) 131 hep-th/9508068].

[11] J. G. Russo and A. A. Tseytlin, "Green-Schwarz superstring action in a curved magnetic Ramond-Ramond background," JHEP9804 (1998) 014 [hep-th/9804076].

[12] R. C. Myers and M. J. Perry, "Black Holes In Higher Dimensional Space-Times," Annals Phys. 172 (1986) 304.

[13] E. Witten, "Instability Of The Kaluza-Klein Vacuum", Nucl. Phys. B195 (1982) 481.

[14] C. G. Callan and J. M. Maldacena, "Brane Dynamics from the Born-Infeld Action", Nucl. Phys. B513 (1998) 198, hep-th/9708147.

[15] M. Fabinger and P. Horava, "Casimir Effect between World-branes in herterotic Mtheory", hep-th/0002073.

[16] K. G. Savvidy, "Brane death via Born-Infeld string," hep-th/9810163.

[17] M. Atiyah, J. Maldacena and C. Vafa, "An M-theory flop as a large n duality," hepth/0011256.

[18] J. Maldacena, "The large N limit of superconformal field theories and supergravity," Adv. Theor. Math. Phys. 2, 231 (1998) [hep-th/9711200]. 
[19] I. R. Klebanov and A. A. Tseytlin, "D-branes and dual gauge theories in type 0 strings," Nucl. Phys. B 546 (1999) 155 [hep-th/9811035].

[20] A. A. Tseytlin and K. Zarembo, "Effective potential in non-supersymmetric SU(N) x SU(N) gauge theory and interactions of type 0 D3-branes," Phys. Lett. B 457 (1999) 77 hep-th/9902095].

[21] I. R. Klebanov, "Tachyon stabilization in the AdS/CFT correspondence," Phys. Lett. B 466 (1999) 166 hep-th/9906220.

[22] A. M. Polyakov, "The wall of the cave," Int. J. Mod. Phys. A 14 (1999) 645 hepth/9809057.

[23] A. Adams and E. Silverstein," Closed String Tachyons, AdS/CFT and Large N QCD", hep-th/0103220.

[24] L. Motl, private communication.

[25] D. Garfinkle and A. Strominger, "Semiclassical Wheeler wormhole production", Phys. Lett. B256 (1991) 146.

[26] D. Garfinkle, S. B. Giddings and A. Strominger, "Entropy in black hole pair production", Phys. Rev. D49 (1994) 958 [gr-qc/9306023].

[27] P. M. Saffin, "Gravitating fluxbranes," gr-qc/0104014. 